\title{
Addressing Patient Acuity and Nurse Staffing Issues in the Acute Care Setting: A Review of the Literature
}

\author{
Tanya Sobaski ${ }^{1}$ \\ ${ }^{1}$ Family Nurse Practitioner, Southwestern Medical Clinic, Bridgman, Michigan, USA \\ Correspondence: Samuel P. Abraham, Associate Professor of Nursing, 1001 Bethel Circle, Bethel College School \\ of Nursing, Mishawaka, Indiana, 46545, USA.
}

Received: July 5, 2018

doi:10.20849/ijsn.v3i3.431

Accepted: July 20, $2018 \quad$ Online Published: July 30, 2018

URL: https://doi.org/10.20849/ijsn.v3i3.431

\begin{abstract}
Background: Many times there is a misalignment on medical-surgical units nursing assignments that do not provide equitable distribution of the patient needs for the unit.

Purpose: The purpose of the literature review was to identify resources that address patient acuity and nurse staffing issues in the acute care setting.

Method: A literature review using the EBSCOhost health search engine, which included databases from Cumulative Index to Nursing and Allied Health Literature (CINAHL), Google Scholar, and ProQuest.

Results: The future of nursing is moving toward staffing based on patient acuity. Chiulli, Thompson, and Reguin-Hartman (2014) developed an acuity tool because it was discovered that there was no appropriate "assessment tool...for [the] medical-surgical patient population" (p. 10). However, nurses are not guaranteed adequate staffing based on acuity regardless of what is determined by use of the tool. Recommended is that further studies need to be conducted using the Chiulli et al. tool.
\end{abstract}

Keywords: acuity tool and medical-surgical unit, failure to rescue and nurse competencies, competencies for nurses and evidence-based practice

\section{Introduction}

Kalisch, Gosselin, and Choi (2012) identified that missed nursing care could be traced back to inadequate nursing resources, including inadequate staffing skill mix. Their study also surmises that sufficient staffing levels, quality teamwork, and good leadership improve patient outcomes. Hospital administrators have been developing an initiative to examine how to reduce the number of resuscitation events that were occurring on medical/surgical units. Aiken, Clarke, Sloane, Sochalski, and Silber (2002) found that nurse staffing had a noticeable effect on patient outcomes and mortality on medical/surgical units. When patient clinical severity and nurse workload indicators are misaligned, patients and nurses suffer. Patient outcomes decline through increased mortality and morbidity. Patients' satisfaction decreases when they feel their needs are not being met. Nurses also experience reduced satisfaction when they are not able to meet the needs of their patients effectively (Aiken et al., 2002). Hospital reimbursement is affected by patient satisfaction. The morale of the nurses can influence patient satisfaction and the morale of the hospital unit. These feelings can affect the retention of nursing staff. The use of an objective acuity rating system that balances the nursing assignments on medical-surgical units leads to the reduction of resuscitation event occurrences (Health Research \& Educational Trust, 2016a). The purpose of the literature review was to identify resources that address patient acuity and nurse staffing issues in the acute care setting.

\section{Background}

Nurse staffing assignments are based on a volume-based distribution of the patient census. Nurses were assigned a group of patients based on an equitable numeric distribution. Using volume-based census staffing does not take into account the acuity of the patient or the competency and workload demanded of the nurse. When the nurse has a high acuity patient or patients that require more care than usual, the nurse may not have the time or ability to recognize changes in the patient's condition. These changes can lead to deterioration in the physiological status leading to failure to rescue by nursing and a resuscitation event (Ashcraft, 2004). 
Assignments are often based on convenience of the location of patient rooms in an area. Patient assignments were made by assigning blocks of rooms to nurses usually in sequential order. Through bad luck or poor planning, patients with high needs are often grouped together. High needs patients increase the acuity for some patient assignments more than others. Imbalanced workloads that do not consider the severity of the patient's illness and the required workload of the nurse can lead to less than optimal care. When the nursing staff is overburdened by the care requirements for multiple patients, the risk of failure to recognize subtle changes or meet all the needs for care is constrained by a lack of time, attention, or awareness.

\section{Review of the Literature}

An initial review of the literature was conducted using the EBSCOhost health search engine, which included databases from Cumulative Index to Nursing and Allied Health Literature (CINAHL), Google Scholar, and ProQuest with the keywords and phrase combinations for "acuity tool and medical-surgical unit," "failure to rescue and nurse competencies," and "competencies for nurses and evidence-based practice." Articles were selected based on relevance to the study and applicability of the setting, staff skill mix, and patient and nurse populations. Articles about acuity tools specific to medical-surgical units were limited. Of the acuity tools found in the research, many were complex and were not aligned with the purpose of the project. Additional articles were included based on references from articles selected, journal subscription articles, recommendations from the project committee members, and position statements about patient safety, acuity tools, and safe nurse staffing.

\subsection{Acuity of Patients in Medical-Surgical Units}

Patients currently placed on medical-surgical units are "vulnerable-as a result of large nurse caseloads, a high percentage of new nurses on staff, and higher levels of patient acuity" (Jones, 2013, p. 38). Some of these patients need more nursing care than is currently possible with these units. Reasons for the misalignment can be due to the complexity of patient care needs, patient acuity, co-morbidities, workload and competency levels of nurses, nurse experience level, failure to identify patient decline and resuscitation events, and lack of knowing when to escalate care. Figure 1 illustrates the multiple factors that need to be addressed regarding patient acuity and nurse staffing in hospital acute care units.

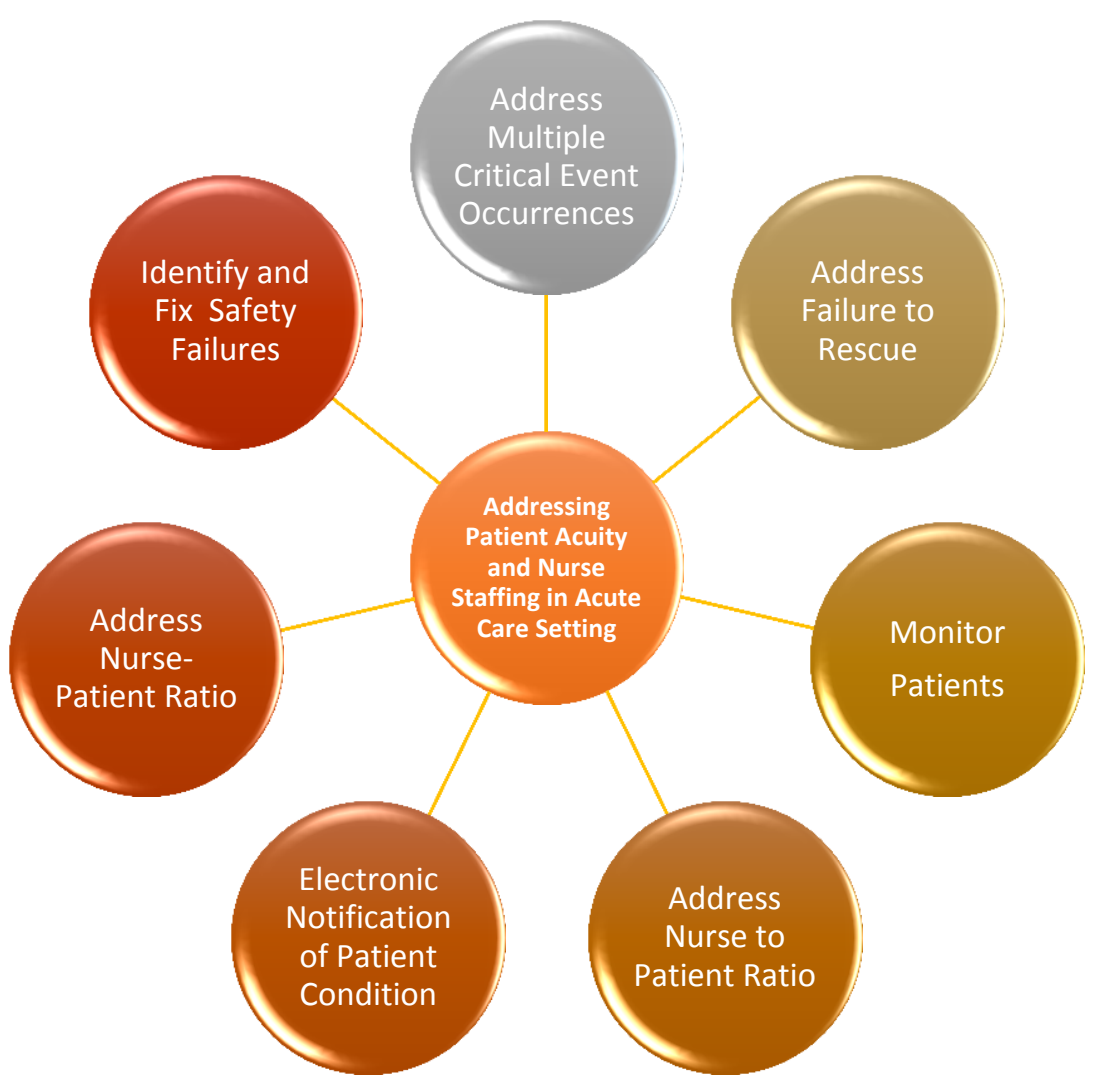

Figure 1. Addressing patient acuity and nurse staffing in hospital acute care units 
Retrospective studies have found a correlation between staffing levels, acuity levels, and patient mortality. When researchers looked at nursing workload indicators, they found patient care indicators for "falls incidence, catheter-associated urinary tract infections, central line-associated bloodstream infections, and pressure ulcers prevalence" all decreased when "staffing was adjusted to account for higher-acuity patients" (O'Keeffe, 2016, pp. 31-32). Data collected by the EHR can be seamlessly adapted to determine the complexity of the patient regarding nursing workload. Researchers suggest balancing facility capabilities with patient needs, mobility, functionality, and physiological and psychosocial needs with nurses' training and skill set (O'Keeffe, 2016).

\subsection{Failure to Rescue}

Inadequate staffing could lead to failure to rescue. Parker (2014) stressed the importance of nurses' being able to "recognize and then react to the antecedent events leading to critical illness and possible FTR [failure to rescue]" (p. 161). Nurses use initiative and analytical decision-making in prompting them to escalate care to avoid a resuscitation event. Recognizing clinical deterioration requires critical thinking by the nurse. Critical thinking is a complex process that requires time, awareness, and recognition to problem-solve and make sound clinical decisions.

Research has demonstrated that measurable physiological changes occur in patients up to 48 hours before cardiopulmonary arrest (Shiloh, Lominadze, Gong, \& Savel, 2016). There are "objective parameters" that, when examined in a trending manner, can predict "physiological dysfunction" (Howard, 2016, p. 14). Failure to rescue (FTR) was a term coined by Silber, Sankey, Krakauer, and Schwartz in 1992 that has become standard terminology in the research literature and is used by the Agency for Health Care Quality as a safety indicator. Failure to rescue as defined by Silber et al. is an unintended complication that occurs in the hospital that increases the risk of death (Hravnak, Schmid, Ott, \& Pinsky, 2010). With patient complexity increasing, lengths of stay shortening, and reimbursement based on quality indicators, one has to examine the cost-effectiveness of nursing hours compared to care provided to patients and health outcomes. Despite legislation mandating staffing ratios, Donaldson and Shapiro (as cited in Twigg et al., 2013) stated that studies have failed to demonstrate "any significant impact on patient safety indicators" (p. 2254).

Changes in patients' physiological status can lead to increased mortality. Failure to rescue requires early recognition and escalation of care. Increased surveillance, awareness, and intervention by nursing staff can increase patient outcomes and reduce mortality (Aiken et al., 2002; Andrews \& Waterman, 2005; Johnston et al., 2015; Twigg et al., 2013).

\subsection{Nurse to Patient Ratio}

Needleman, Buerhaus, Stewart, Zelevinsky, and Mattke (2006) found that while increased registered nurse to patient staffing ratios did not result in cost savings, the benefit for improved patient outcomes was justified. Their research of 799 non-government hospitals in 11 states found that by increasing the nurse staffing ratios, there was a reduction in the lengths of stay, improved patient outcomes, decreased adverse events, and decline in the number of failures to rescue events. The value of the patient population and societal benefit justified the lack of cost-savings. The research by Needleman et al. (2006) highlights additional ways hospitals can "improve quality and patient safety...[such as] equipping hospitals with new technology, investing in training and education, imposing regulations, and increasing nursing staff" (p. 205).

\subsection{Vital Monitoring of Patients}

Respiratory rates have been shown to be an indicator of changes in patient status and are an indicator of physiological deterioration (Andrews \& Waterman, 2005). Improved communication among care providers to report changes in vital sign alerts (VSA) as part of the early warning system (EWS) has been demonstrated to reduce unplanned transfer to a higher level of care and "an increased risk of morbidity and mortality" (Johnston et al., 2015, p. 831; Ashcraft, 2004; Bonnici, Gerry, Wong, Knight, \& Watkinson, 2016; Ghaferi \& Dimick, 2015; Howard, 2016; Jones, 2013). Early Warning Systems are designed to be a preemptive response to decreasing patient morbidity and mortality. However, as Landro (2015) pointed out, "Signs aren't always picked upon or acted upon by staff" (para 3). The triad of vital signs being monitored are common variables that could indicate the risk of a cardiopulmonary arrest (Andrews \& Waterman, 2005).

Andrews and Waterman (2005) used a qualitative study design based on the grounded theory design of conversational interviewing. Their findings were that nurses tended to use subjective, "intuitive knowing," and non-medical terminology that impaired their credibility and professional opinions, which led to not being taken seriously by physicians (p. 478). The recommendation based on the study was that nurses use the EWS as part of 
"a systematic approach to assessing patients who are vulnerable to physiological deterioration" (p. 479). The authors also felt that the EWS improved communication and confidence since it was linked to objective data.

\subsection{Electronic Notification of Patient Condition}

Bonnici et al. (2016) evaluated the implementation of the System for Electronic Notification and Documentation protocol for an escalation of care based on the Agency for Healthcare Research and Quality (AHRQ) Health Information Technology (HIT) Evaluation Toolkit and the Delone \& MeLeans's Model of Information System Success. This study was a four-step wedge design that examined four hospital systems looking at "nursing's recognition and response to signs of deterioration" (Bonnici et al., p. 3) and time until escalation of care or discharge. The findings for the endpoints "proved to be challenging" (p. 7) and were focused on paper versus electronic charting.

\subsection{Identifying and Fixing of Safety Failures}

Johnston et al. (2015) discussed the progression of escalation of care using the Healthcare-Failure-Mode-Effects-Analysis to identify safety failures and develop solutions. Historically, this type of analysis has resulted in the development of safety protocols, such as situation, background, assessment, response/recommendation (SBAR) and the surgical care improvement project (SCIP), which have been shown to reduce and prevent hazardous failures in healthcare. The study identified failure to rescue rates between $29 \%$ and $41 \%$, with "early escalation of care is critical to prevent avoidable harm and improve outcomes" (Johnson et al., 2015 , p. 837). With regards to nursing, the study identified seven failures in the escalation of care that can result in resuscitation events. Many of these were the result of communication issues between the patient and nurse, and the nurse and the medical residents and doctors, as well as vital sign documentation issues. "Participants felt that understaffing was the principle cause of all these failures" (Johnston et al., 2015, p. 834). The recommendations were to "increase nurse to patient ratios," "use electronic vital sign recording and documentation systems," "remove hierarchical barriers through the development of an improved escalation protocol," and "[increase] use of Smartphone technology" (p. 834).

\subsection{Addressing Multiple Critical Event Occurrences}

Both Needleman et al. (2006) and Hravnak et al. (2010) discussed the need to identify complications which can result in adverse events. There is a multi-pronged responsibility on the part of the hospital system to have measures in place to help recognize and respond to the deterioration of the patient's physiological status. Nursing must be vigilant in identifying and intervening when physiological changes occur and implement interventions to correct the changes and prevent deterioration.

A study of critical illness events on medical-surgical units at the University of Chicago from 2009 to 2013 found that when there is a critical event (i.e., resuscitation event, ICU transfer, or death) on a medical-surgical unit, there is an increased risk of another event occurring on that same unit within the next 6 hours. This study was an observational study of a 13-unit cohort of medical-surgical units with an average patient-nurse ratio of 4:1, with a charge nurse, attending physician and two to three residents. Each unit had approximately 20 beds. The odds adjusted risk ratio was 1.18 for one event and 1.53 for more than one event. The absolute adjusted risk demonstrated an increase of 1.9 occurrence events (Volchenboum \& Mayampurath, 2016).

The Health Information Technology for Economic and Clinical Health (HITECH) Act, which is part of the American Recovery and Reinvestment Act of 2009 was designed to "improve workflow and documentation to help make care safer and more efficient" (Weiner, Fowles, \& Chan, 2012, para. 3). The use of HIT and EHR can be used to measure quality measures, known as "e-QM." Implementation of a clinical decision support system is used to support nursing surveillance and use of "actionable targets" (Lewis, 2016, para 14; McGee, 2016). CDS systems and nurse-sensitive outcomes have been used to prevent FTR events such as sepsis, septic shock, hypotension, gastrointestinal bleed, respiratory failure, and cardiac arrest (Twigg et al., 2013).

The American Heart Association (AHA) utilizes a voluntary reporting system for cardiac arrest patients. Reporting is encouraged to add to the data and development of evidence-based care. Participating hospitals report both in and out of hospital resuscitation events. The data that is collected is used to provide feedback on their resuscitation practice and patient outcomes, as well as to develop new evidence-based guidelines.

Three descriptive areas look specifically at the histogram of patients who deteriorate on medical-surgical units. These include the Medical Emergency Team (MET), Acute Respiratory Compromise (ARC), and Cardiopulmonary Arrest (CPA). These are meant to be a step-wise increase in the level of care based on the patient's physiological status and the nurse's ability to manage the changing patient hemodynamic status. 


\subsection{Staffing Laws and Regulations}

The American Nurses Association ([ANA], 2015) has been working on implementing legislation that balances staffing to provide safe care to hospitalized patients. The goal is to have appropriate staffing for the level of care to improve patient outcomes and satisfaction, reduce errors, improve staff morale and retention, and make the delivery of healthcare more cost-effective. There are currently 14 states that have staffing laws and regulations in place. There is variance between states regarding regulations. Since 1999, California has had a mandated nurse to patient ratio that must be maintained at all times. Other states require that the Chief Nursing Officer (CNO) use collaboration to develop staffing plans. Five states require public reporting of staffing levels (ANA, 2015). While ratios are important, the acuity level between patients on the same unit can vary tremendously.

\subsection{Acuity Measurement Tools}

Oh and Seo (2008) determined that numerous acuity measurement tools had been developed to help determine "disease severity and ... to predict outcomes, especially mortality" (p. 50). Benner (1984) and Johnston et al. (2015) demonstrated that acuity measurement tools help with identification; they do not provide the nursing staff with support for recognizing and acting on subtle changes that indicate an escalation of care. Benner (1984) discussed the importance of vigilance in recognition of acuity in patient safety. The nurse is expected to manage patients to keep them from life-threatening situations.

Workloads that are equitable improve nursing care, which in turn, improves patient outcomes. Kidd, Grove, Kaiser, Swoboda, and Taylor, (2014) discovered that acuity tools have been shown to decrease hospital expenses and allow "high-quality nursing care" (p. 2). It was also found that nurses "add value... and should be involved in assessing their own workloads and making decisions about resources" (p. 2). "Patient characteristics drive nurse competencies" (Edwards, 1999, p. 1). Providing nurses with autonomy and professional independence improves ownership of their practice, enhances job satisfaction, and promotes advocacy for patients.

Chiulli et al. (2014) developed an acuity tool because it was discovered that there was no appropriate "assessment tool...for [the] medical-surgical patient population" (p. 10). The acuity tool "incorporates clinical severity and nurse workload indicators to determine acuity and is used to make patient assignments in alignment with appropriate skill mix and staffing ratios" (p. 12). The researchers worked with staff and managers to identify assessments that recognized that acuity levels were different for individual patients. Through trialing the acuity tool, the nurses were "able to have an objective tool to use in assessing patient acuity to provide safe care, adjust staffing ratios, and balance unit workload" (p. 12).

The adverse event triggers can be changes in vital signs, changes in neurological status, or abnormal laboratory results that have been determined to be markers in physiological deterioration. The Adverse Event Trigger Tool is a retrospective, root-cause analysis of events that had "unintended consequences of medical care ...preventable or not....which caused harm" (Griffin \& Resar, 2009, p. 8). The recognition of patient deterioration improves patient safety and trigger tools are used to track adverse events. Patient morbidity and mortality are indicators used as quality measures by the Center for Medicare and Medicaid Services (CMS), the AHRQ, and the Institute of Medicine (IOM). These agencies have continued the call for quality nursing practice to prevent patient harm.

\subsection{Determining Nurse Competencies}

Sherwood and Zomorodi (2014) discussed the implementation of Quality and Safety Education for Nurses (QSEN) core competencies in providing safe, patient-centered care. Utilizing the QSEN knowledge, skills, and attitude (KSA) competencies, they operationalized the Team Strategies and Tools to Enhance Performance and Patient Safety (TeamSTEPPS) curriculum from AHRQ. The strategies are designed around improved communication to increase interdisciplinary and intradisciplinary collaboration.

The strategies in the TeamSTEPPS curriculum are

- SBAR: Situation, background, assessment, and recommendation,

- CUS "C-I am concerned, U- I am uncomfortable, S - I think this is a safety issue, if no action, next step is to go up the chain of command."

- Check back or read back, repeating important communication.

- Plan of care briefings determining the following: "What is the most important thing this patient needs? What are the safety issues? What are the benchmarks and/or evidence for the care interventions?"

- Huddles to "problem solve or clarify strategy, get everyone on the same page" by asking, "What is the priority? What else could it be? What could we do differently? What was done well?" 
- Debriefing strategies such as asking, "What did not go well? What could be done differently?" (AHRQ, as cited in Sherwood \& Zomorodi, 2014, p. 19, Table 1).

Determining nurse competencies have been a topic of discussion for decades, many using Benner's Novice to Expert theoretical framework (Wangensteen, Johansson, \& Nordstrom, 2014). There have been numerous tools and methods of evaluating competency in nursing; however, "repeated use of instruments for measuring nurse competence has rarely been reported" (Wangensteen et al., 2014). The Nurse Competency Scale has been used internationally to measure new graduate competence, but there has been dissension in a common definition and in establishing validity and reliability. Corwin et al. (2008) demonstrated a relationship between competencies between the Australian National Competencies and the Nurse Competency Standards for new graduates.

Engelmann, Brady, Larson, Perkins, and Schulz (2012), working with the National League for Nursing (NLN) in response to the IOM's reports (To Err Is Human, 2000; Crossing the Quality Chasm, 2001; The Future of Nursing: Leading Change, Advancing Health, 2010) calling for reform of competencies in nursing and nursing education, sought to "design competencies that address the affective, cognitive, and psychomotor domains essential for nursing practice" (para. 1). The result of their work was the NLN Education and Competencies Model (EMC), which serves to guide the incorporation of implementing the NLN "core values, integrating concepts, and program outcomes" (para. 2).

Conceptually, the EMC combine to develop core competencies in nursing. The core concepts, defined by the NLN were expanded and include "Caring, Diversity, Excellence, Integrity, Ethics, Holism, and Patient Centeredness" (Engelmann et al., 2012, para. 3). The Education and Competencies Models integrates concepts derived from the Educating Nurses: A Call for Radial Transformation, 2010, report calling for nursing education to be built on the framework of "Context and Environment, Knowledge and Science, Personal/Professional Development, Quality and Safety, Relationship-Centered Care, and Teamwork" (Engelmann et al., 2012, para. 3). The program outcome attributes of the graduate nurse include "Human Flourishing, Nursing Judgment, Professional Identity, and Spirit of Inquiry" (Engelmann et al., 2012, para. 3).

It is more challenging to assess competencies for seasoned registered nurses. The literature for determining the competency of nursing has demonstrated that self-evaluation is a valid and reliable way to demonstrate nursing. Other ways are through "observation of practice....learning packages....continuing professional development" (Royal Children's Hospital, n.d. para. 5).

Hwang (2015) developed and tested a patient care competency (PCC) scale based on KSAs based on QSEN competencies. The QSEN project focuses on patient-centered care and the relationship the nurse has with the patient. The study was based on a 17 -item questionnaire, with nurses $(n=577)$ rating "their competencies on a Likert scale of $1=$ minimal... [to] 5 = excellent" (p. 45). The scale was able to demonstrate "good psychometric properties to assess nurses' competency for patient-centered care" (p. 47). This scale can be used to "assess nurses' competency for patient-centered care in hospital setting.... [demonstrate] variations in total PCC scores according to individual and organizational characteristics... Determine factors related to patient-centered care.... [and] used for evaluation of educational interventions...to improve PCC" (p. 50).

Melnyk, Gallager-Ford, Long, and Fineout-Overholt (2014) developed evidence-based practice (EBP) competencies for both registered nurses and advanced practice nurses using a Delphi consensus-building survey model for use in acute care health care settings. The findings were determining set competencies for registered and advanced practice nurses. The authors determined that the findings could be used to incorporate the results into "healthcare systems expectations, orientations, job descriptions, performance appraisals, and clinical ladder promotions ... driving higher quality, reliability, and consistency of healthcare as well as reduc[ing] costs" (Abstract).

\section{Conclusion}

The review of the literature indicated several factors associated with patient acuity and staffing. The factors include failure to rescue because of inadequate staffing, lack of competency in addressing the problem, and lack of tools to measure acuity. The future of nursing is moving toward staffing based on patient acuity. However, nurses are not guaranteed adequate staffing based on acuity regardless of what is determined by use of the tool. Finance, support from administrators, adequate reimbursement, and availability of staffing, are some of the determining factors. Continued research is essential in balancing facility capabilities and patient needs with nurses' training and skill. 


\section{References}

Aiken, L.H., Clarke, S.P., Sloane, D.M., Sochalski, J., \& Silber, J.H. (2002). Hospital nurse staffing and patient mortality, nurse burnout, and job dissatisfaction. JAMA, 288(16). https://doi.org/10.1001/jama.288.16.1987

ANA. American Nurses Association. (2015). Safe Staffing: The Registered Nurse Safe Staffing Act (H.R. 876/S. 58). Retrieved from www.anapoliticalpower.org

Andrews, T., \& Waterman, H. (2005). Packaging: A grounded theory of how to report physiological deterioration effectively. Journal of Advanced Nursing, 52(5), 473-481. https://doi.org/10.1111/j.1365-2648.2005.03615.x

Ashcraft, A.S. (2004). Differentiating between pre-arrest and failure-to-rescue. MedSurg Nursing, 13(4), 211-215.

Benner, P. (1984). From novice to expert: Excellence and power in clinical nursing practice. Menlo, CA: Addison-Wesley.

Bonnici, T., Gerry, S., Wong, D., Knight, J., \& Watkinson, P. (2016). Evaluation of the effects of implementing an electronic early warning score system: Protocol for a stepped wedge study. BioMed Central Medical Informatics and Decision Making, 16(19). https://doi.org/10.1186/s12911-016-0257-8

Chiulli, K., Thompson, J., \& Reguin-Hartman, K.L. (2014). Development and implementation of a Patient Acuity Tool for a Medical-Surgical Unit. Academy of Medical-Surgical Nurses, 23(2), 9-12.

Corwin, L.S., Hengstberger-Sims, C., Eagar, S.C., Gregory, L., Andrew, S., \& Rolley, J. (2008). Competency measurements: testing convergent validity for two measures. Journal of Advanced Nursing, 64(3). https://doi.org/10.1111/j.1365-2648.2008.04774.x

Edwards, D.F. (1999). The Synergy Model: Linking patient needs to nurse competencies. Critical Care Nurse, 19(1). Retrieved from http://www.aacn.org/wd/certifications /content/synpract2.pcms?menu

Engelmann, L., Brady, M., Larson, J., Perkins, I., \& Schulz, C. (2012). Transforming nursing education: The NLN education competencies model. Nursing Education Perspectives. Retrieved from http://www.readperiodicals.com/201205/2697658441.html

Ghaferi, A.A., \& Dimick, J.B. (2015). Understanding failure to rescue and improving safety culture. Annals of Surgery, 261(5), 839-840. https://doi.org/10.1097/SLA.0000000000001135

Griffin, F.A., \& Resar, R.K. (2009). IHI global trigger tool for measuring adverse events. Retrieved from http://www.ihi.org/resources/Pages/Tools/ IHIGlobalTriggerToolforMeasuringAEs.aspx

Health Research \& Educational Trust. (2016a). Failure to Rescue Change Package: 2016. Chicago, IL: Health Research \& Educational Trust. Retrieved from www.hret-hen.org

Howard, D. (2016). Using the Military Medical Acuity Model to guide patient care. Nursing2016, 46(1), 14-17. https://doi.org/10.1097/01.NURSE.0000475493.62149.71

Hravnak, M., Schmid, A., Ott, L., \& Pinsky, M.R. (2010). Causes of failure to rescue. In M. A. DiVita, K. Hillman, \& R. Bellomo (Eds.), Textbook of Rapid Response Systems: Concept and Implementation (pp. 141-150). Philadelphia, PA: Springer.

Hwang, J. (2015). The development and testing of a patient-centered care competency scale for hospital nurses. International Journal of Nursing Practice, 21, 43-51. https://doi.org/10.1111/ijn.12220

Johnston, M., Arora, S., Anderson, O., King, D., Behar, N., \& Darzi, A. (2015). Escalation of care in surgery: A systematic risk assessment to prevent avoidable harm in hospitalized patients. Annals of Surgery, 261, 831-838. https://doi.org/10.1097/SLA.0000000000000762

Jones, B.G. (2013). Developing a vital sign alert system. Amercian Journal of Nursing, 113(8), 36-44. https://doi.org/10.1097/01.NAJ.0000432962.33881.65

Kalisch, B.J., Gosselin, K., \& Choi, S.H. (2012). A comparison of patient care units with high versus low levels of missed nursing care. Health Care Management Review, 37(4), 320-328.

Kidd, M., Grove, K., Kaiser, M., Swoboda, B., \& Taylor, A. (2014). A new patient-acuity tool promotes equitable nurse-patient assignments. AmericanNurseToday.com, 9(3), 1-4.

Landro, L. (2015). Hospitals find new ways to monitor patients 24/7. Wall Street Journal. Retrieved from http://webreprints.djreprints.com/3670891403610.html 
Lewis, R. (2016). Better nursing environments linked to greater surgical value. Medscape. Retrieved from http://www.medscape.com/viewarticle/857550?nlid=97686_2822\&src =wnl_edit_medp_nursing

McGee, E. (2016). Failure to rescue. Advance Healthcare Network. Retrieved from http://nursing.advanceweb.com/Editorial/Content/PrintFriendly.aspx?CC=195828

Melnyk, B.M., Gallagher-Ford, L., Long, L.E., \& Fineout-Overholt, E. (2014). The establishment of evidence-based practice competencies for practicing registered nurses and advanced practice nurses in real-world clinical settings: Proficiencies to improve healthcare quality, reliability, patient outcomes, and costs. Worldviews on Evidence-Based Nursing, 11(1), 5-15. https://doi.org/10.1111/wvn.12021

Needleman, J., Buerhaus, P.I., Stewart, M., Zelevinsky, K., \& Mattke, S. (2006). Nurse staffing in hospitals: Is there a business case for quality?. Health Affairs, 25(1), 204-211. https://doi.org/10.1377/hlthaff.25.1.204

Oh, H., \& Seo, W. (2008). Validity for the critical patient's severity classification system developed by the Korean Clinical Nurse Association. Australian Journal of Advanced Nursing, 25(3), 49-57.

O'Keeffe, M. (2016, September). Practical steps for applying acuity-based staffing. American Nurse Today, 11(9). Retrieved from https://www.americannursetoday.com/practical-steps-applying-acuity-based-staffing/

Parker, C.G. (2014). Decision-making models used by medical-surgical nurses to activate rapid response teams. MedSurg Nursing, 23(3), 159-164.

Royal Children's Hospital (n.d.). Nursing competency framework: Demonstration of competence. Retrieved from http://www.rch.org.au/mcpc/nursing_competency_framework/

Sherwood, G., \& Zomorodi, M. (2014). A new mindset for quality and safety: The QSEN competencies redefine nurses' roles in practice. Nephrology Nursing Journal, 4l(1).

Shiloh, A.L., Lominadze, G., Gong, M.N., \& Savel, R.H. (2016). Early warning/track-and-trigger systems to detect deterioration and improve outcomes in hospitalized patients. Semin Respir Crit Care Med 2016, 37(01). 88-95. https://doi.org/10.1055/s-0035-1570352

Silber, J.H., Sankey, V., Krakauer, H., \& Schwartz, S. (1992). Hospital and patient characteristics associated with death after surgery: A study of adverse occurrence and failure to rescue. Medical Care, 30(7). Retrieved from http://journals.lww.com/lww-medicalcare/Abstract/1992/07000/Hospital_and_Patient_Characteristics_Asso ciated.4.aspx

Twigg, D.E., Geelhoed, E.A., Bremner, A.P., \& Duffield, C.M. (2013). The economic benefits of increased levels of nursing care in the hospital setting. Journal of Advanced Nursing, 69(10), 2253-2261. https://doi.org/10.1111/jan.12109

Volchenboum, S.L., \& Mayampurath, A. (2016). Association between in-hospital critical illness events and outcomes in patients on the same ward. JAMA, Journal of the American Medical Association, 316(24), 2674-2675. https://doi.org/10.1001/jama.2016.15505

Wangensteen, S., Johansson, I.S., \& Nordstrom, G. (2014). Nurse competence scale-Psychometric testing in a Norwegian context. Nurse Education in Practice, 15, 22-29. https://doi.org/10.1016/j.nepr.2014.11.007

Weiner, J.P., Fowles, J.B., \& Chan, K.S. (2012). New paradigms for measuring clinical performance using electronic health records. International Journal of Quality Health Care, 24(3), 200-205. Retrieved from www.medscape.com/viewarticle/764288_print

\section{Copyrights}

Copyright for this article is retained by the author(s), with first publication rights granted to the journal.

This is an open-access article distributed under the terms and conditions of the Creative Commons Attribution license (http://creativecommons.org/licenses/by/4.0/). 